\title{
FLORA PRESENTE EN LAS ÁREAS VERDES DEL CENTRO NUCLEAR "OSCAR MIRO QUESADA DE LA GUERRA”, CARABAYLLO, LIMA
}

\author{
TAXONOMIC IDENTIFICATION OF FLORA LOCATEDIN THE GREEN AREA SOF THE \\ NUCLEAR CENTER “OSCAR MIRO QUESADA DE LA GUERRA”, CARABAYLLO, LIMA
}

\author{
${ }^{1}$ Juan Agapito, ${ }^{1,2 *}$ Gladys Tello, ${ }^{1,3}$ Yuriko Ortega y ${ }^{4}$ Marco Espinoza
}

\begin{abstract}
Resumen
Un estudio llevado acabo entre julio y noviembre de 2014 sobre la flora presente en las áreas verdes dentro de las 16 hectáreas del Centro Nuclear "Oscar Miró Quesada de la Guerra (RACSO)", Carabayllo, Lima, ha permitido la identificación de un total de 70 especies de plantas vasculares distribuidas en 39 familias y 62 géneros. Las familias más representativas fueron: Fabaceae, Euphorbiaceae y Solanaceae con 8 (11\%), 5 (7\%) y 4 (6 \%) especies respectivamente. Además, se indican los géneros y especies endémicas del Perú registradas en la zona estudiada. Este trabajo es un esfuerzo inicial para promover el conocimiento de las especies biológicas que viven en la zona del emplazamiento del Reactor RP-10 y poner en valor la biodiversidad presente en este lugar.
\end{abstract}

Palabras claves: Plantas vasculares, Especies, Flora, Centro Nuclear RACSO, Biodiversidad, Áreas verdes.

\begin{abstract}
A study conducted from July to November 2014, on the flora located in the green areas inside the 16 hectares of the Nuclear Centre "Oscar Miró Quesada de la Guerra (RACSO)", Carabayllo, Lima, has permitted the identification of a total amount of 70 species of vascular plants distributed in 39 families and 62 genera. The most representative families were Fabaceae, Euphorbiaceae and Solanaceae with $8(11 \%), 5(7 \%)$ and 4 (6\%) species respectively. Besides, the genera and species endemic in Perú founded in the studied area are indicated. This work is an initial effort to promote the knowledge of biological species living in the influence zone of RP-10 reactor and to highlight the meaning of biodiversity present in this place.
\end{abstract}

Key words: Vascular plants, species, flora, nuclear center, biodiversity, green areas.

\section{Introducción}

Las áreas verdes ubicadas en la ciudad y centros de trabajo generan beneficios en sus habitantes, mejora la calidad del aire reduciendo los niveles de polución, influye en el grado de radiación solar y movimiento del viento, regulan la temperatura y reducción de los efectos de las islas de calor, mediante sombreamiento y evapotranspiración [1].

Asimismo, favorecen la presencia de especies de flora y fauna nativa, contribuyendo a la conectividad de los ecosistemas silvestres y estableciendo una continuidad entre los espacios silvestres, rurales y urbanos [2]. Por otro lado, genera la disminución del estrés mejorando el estado de ánimo en las personas.

El Centro Nuclear "Oscar Miró Quesada de la Guerra (RACSO)” del Instituto Peruano de Energía Nuclear
(IPEN), se encuentra ubicado en el Distrito de Carabayllo, Provincia de Lima, Perú. Geográficamente se asienta sobre la costa y forma parte de la cadena de lomas costeras del Perú. Desde la inauguración del Centro Nuclear en 1988 la institución viene desarrollando dentro de su zona de influencia la formación de áreas verdes sobre un terreno particularmente árido, donde inicialmente no había poca vegetación como cactáceas y tillandsias grises (mayormente litófitas) que crecen en los cerros que rodean dicho emplazamiento. A lo largo del tiempo se han venido haciendo trabajos de formación de suelo apto para el crecimiento de plantas mediante técnicas de habilitación agrícola que en más de 25 años han dado como resultado una zona verde de más de 10 hectáreas que proyecta hoy en día la imagen de un verdadero oasis en medio de la sequedad de este territorio (Figura1).

\footnotetext{
${ }^{1}$ Laboratorio de Biología Molecular. Instituto Peruano de Energía Nuclear. Av. Canadá 1470, Lima, Perú.

${ }_{3}^{2}$ Facultad de Ciencias -Biología, Universidad Nacional Agraria la Molina Av. La Molina S/N, Lima, Perú. Email: tcgladys@gmail.com Facultad de Ciencias Biológicas, Universidad Nacional Mayor de San Marcos, Av. Venezuela. S/N, Lima, Perú.

${ }^{4}$ Laboratorio de Citogenética y Radiobiología. Instituto Peruano de Energía Nuclear. Centro Nuclear "Oscar Miró Quesada de la Guerra", km 13, Av. Carabayllo, Lima, Perú.
} 


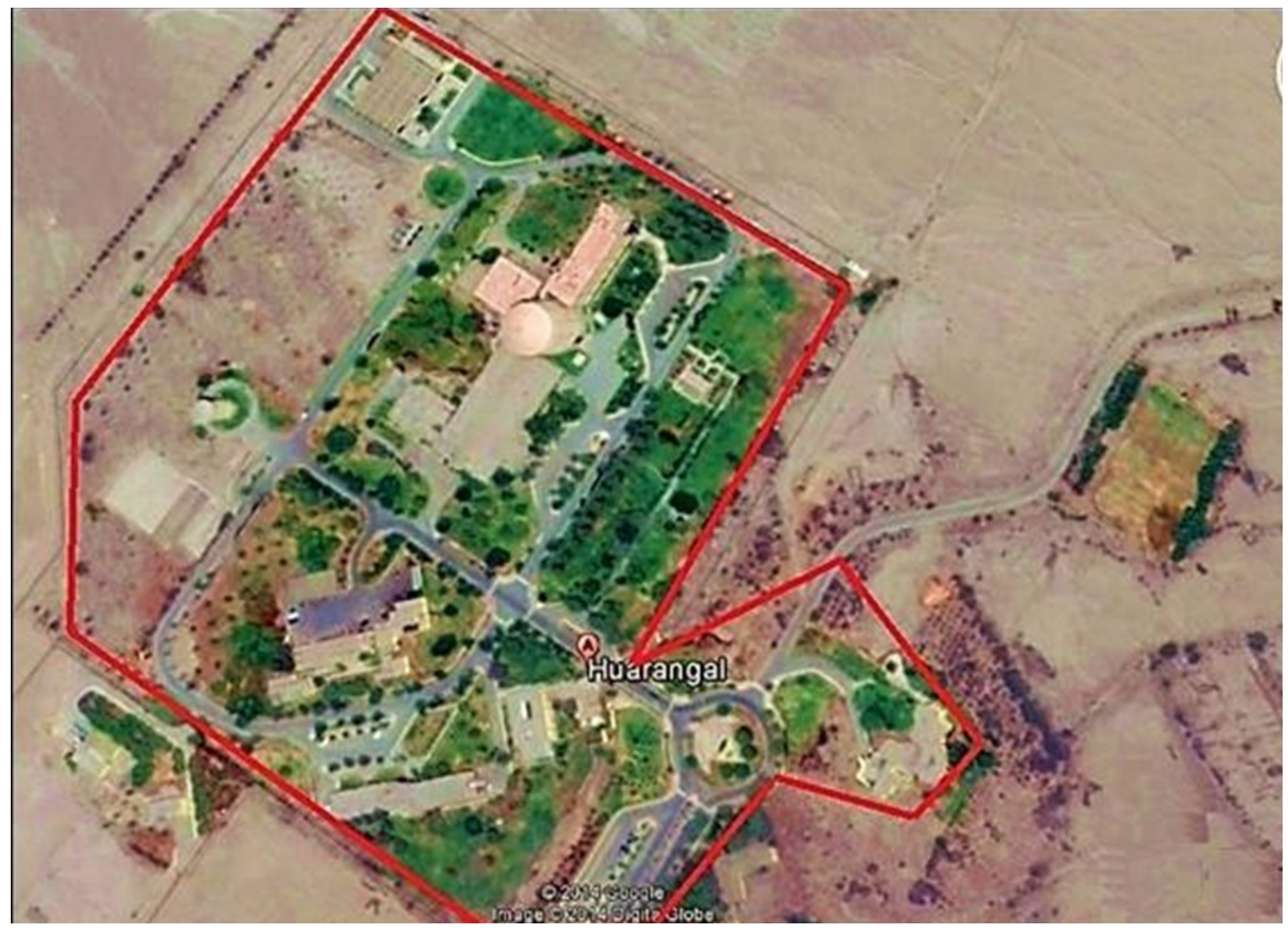

Figura 1. Zona de muestreo dentro de las 16 hectáreas del Centro Nuclear “Oscar Miró Quesada de la Guerra (RACSO)" (líneas rojas).Fuente: Imagen obtenida mediante Google Map.

Según el Estudio de la Zona de Emplazamiento del Centro Nuclear de Investigaciones del Perú [4], la zona de interés para el presente estudio (16 ha.) queda dentro de la denominada Pampa de Huarangal que se describe como una pequeña llanura formada por la conjunción de conos aluvionales procedentes de los cauces de tres quebradas, con una pendiente promedio de $5 \%$ y una superficie cubierta por clastos aluvionales que dejan translucir antiguos drenajes naturales hacia la cuenca del río Chillón, que está a $2 \mathrm{~km}$, siendo un río de escasa corriente en la mayor parte del año. Es, por ende, un terreno árido y pedregoso.

La vegetación que crece en las áreas verdes que rodean al Reactor RP-10 es vegetación totalmente introducida durante los años de funcionamiento del Centro Nuclear. Desde los árboles más grandes hasta los pastos y hierbas han sido sembrados por la mano del personal de jardinería bajo la supervisión de profesionales entendidos en asuntos agronómicos. El mantenimiento de las áreas verdes se ha logrado gracias a la disposición permanente de agua que es extraída del subsuelo y utilizada para el regadío de los jardines. El suelo preparado con tierra proveniente de las zonas agrícolas cercanas ha sido mezclado con el suelo original y periódicamente abonado con estiércol animal y abono químico.
De esta manera las áreas verdes del Centro Nuclear crecieron y acogieron a muchas especies (muchas de las cuales aún no han sido identificadas), creándose un lugar grato para ser visitado y contrastando con el agreste paisaje de fondo. Asimismo, muestra una diversidad de especies que vista de cerca resulta interesante y curiosa en un lugar originalmente tan árido como las Pampas de Huarangal. El presente trabajo busca promover en el trabajador, visitante y público en general el interés por la biodiversidad presente en las 16 hectáreas del emplazamiento del Centro Nuclear y el conocimiento de las especies vegetales presentes, algunas nativas del Perú otras son originarias de otras regiones del mundo.

\section{Metodología Experimental}

\section{1 Área del estudio}

El Centro Nuclear "Oscar Miro Quesada de la Guerra (RACSO)"se ubica en el Departamento de Lima, Provincia de Lima, Distrito de Carabayllo, al norte de Lima. Registra una altitud de $400 \mathrm{msnm}$, está ubicado entre las coordenadas $77^{\circ} 00^{\prime} 40^{\prime \prime}$ de longitud oeste y $11^{\circ}$ 47'50" de latitud sur [3]. La precipitación pluvial es de $50 \mathrm{~mm}$ anuales y las temperaturas, máxima y mínima son entre $30^{\circ} \mathrm{C} \mathrm{y} 16^{\circ} \mathrm{C}$, respectivamente. 
En la Figura 1 se ha demarcado con una línea roja la zona dentro de la cual se procedió a realizar las observaciones de campo y las colectas de material biológico para la identificación taxonómica. Durante las salidas se procedió a tomar fotografías de los especímenes escogidos para el estudio de cada especie.

\subsection{Trabajo de campo}

Se realizaron 4 jornadas de trabajo de campo dentro de las 16 hectáreas para hacer las observaciones directas de las plantas, la elección de los especímenes de estudio, el registro de sus características, la colecta de partes de estos especímenes para facilitar la identificación taxonómica y finalmente la toma de fotografías de los individuos estudiados. Las muestras fueron recolectadas y trasladas al Laboratorio de Biología Molecular del IPEN y al Herbario MOL-Weberbauer de la Universidad Nacional Agraria La Molina, para su identificación.2.3

\subsection{Clasificación taxonómica:}

Se colectaron muestras botánicas con presencia de flores y frutos las cuales fueron prensadas, codificadas y etiquetadas en campo [5]; para posteriormente ser trasladadas al Herbario MOL-Weberbauer de la Universidad Nacional Agraria La Molina y ser identificadas. Asimismo, se anotaron en la libreta de campo datos característicos de la planta como: Estado fenológico, ubicación y descripción de su hábitat.

Para la identificación de las especies se utilizaron claves taxonómicas y los datos registrados por las diversas técnicas en campo.

\subsection{Procesamiento de los datos}

Los datos de las muestras colectadas así como el material fotográfico fueron procesados en la computadora del Laboratorio de Biología Molecular del IPEN, permitiendo realizar un listado de las familias y especies de plantas colectadas.

\subsection{Determinación taxonómica}

Se realizaron visitas al Herbario MOL- Weberbauer de la UNALM así como el uso de la bibliografía $[6,7,8,9,10]$ para identificar las plantas colectadas.

\subsection{Fichas botánicas}

Se elaboraron fichas botánicas conteniendo información sobre el nombre común, nombre científico, familia a la que pertenece, origen, distribución y descripción botánica de cada ejemplar. Todo esto acompañado de un registro fotográfico, que permite la divulgación científica de $\mathrm{su}$ contenido. Las fichas fueron colocadas en carteles y ubicadas dentro de las áreas verdes del Centro Nuclear de Huarangal (Figura 2).

\section{Resultados}

Se identificaron un total de 70 especies de plantas vasculares distribuidas en 39 familias y 62 géneros (Tabla 1). Siendo las familias más representativas Fabaceae (11\%), seguido de Euphorbiaceae (7\%), Solanaceae (6\%). Arecaceae, Moraceae y Rosaceae $(4.29 \%$ cada una de ellas).

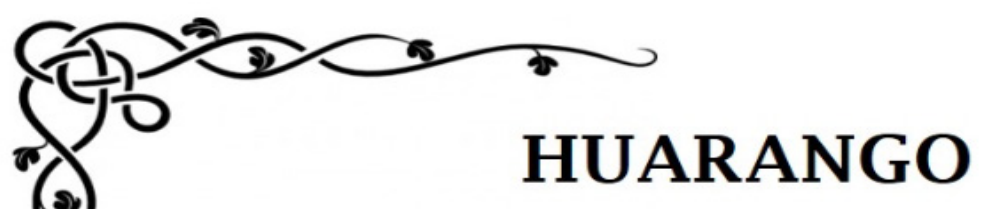

Nombre común:

Huarango, faique, espino, taque

Nombre científico:

Acacia macracantha

- Familia:

FABACEAE

Origen/Distribución:

Perú y Ecuador / Lugares semiáridos en valles costeños e interandinos. Descripción:

Árbol espinoso. Flores en pompones amarillos. Fruto leguminoso pequeño enroscado y aplanado de color negromate.

NATIVA

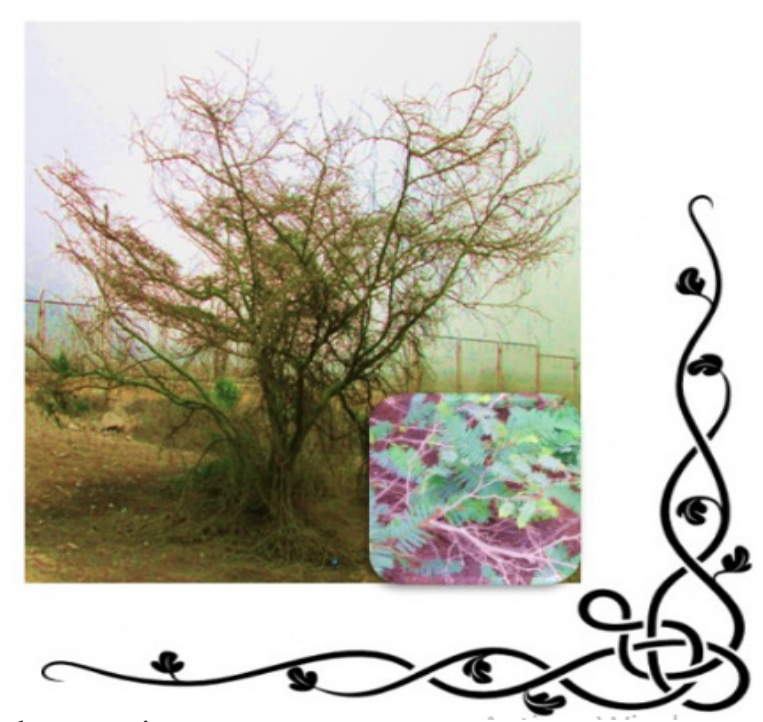

Figura 2. Modelo de ficha botánica y descripción taxonómica de las especies. 
Tabla1. Lista de especies registradas en las áreas verdes del Centro Nuclear "Oscar Miró Quesada de la Guerra (RACSO)", Carabayllo, Lima.

\begin{tabular}{|c|c|c|c|}
\hline $\mathbf{N}^{\circ}$ & Familia & Nombre científico & Nombre común \\
\hline 1 & Aizoaceae & Mesembryanthemum cordifolium L. f. & rocío, escarcha \\
\hline 2 & Anacardiaceae & Schinus molle L. & $\begin{array}{l}\text { molle serrano, árbol de la pimienta, árbol } \\
\text { de la vida }\end{array}$ \\
\hline 3 & Anacardiaceae & Schinus terebinthifolius Raddi & molle costeño \\
\hline 4 & Annonaceae & Annona cherimola Mill. & chirimoya \\
\hline 5 & Annonaceae & Annona muricata L. & guanábana \\
\hline 6 & Apocynaceae & Catharanthus roseus (L.) G.Don & isabelita, vinca rosa \\
\hline 7 & Apocynaceae & Nerium oleander L. & laurel rosa, adelfa \\
\hline 8 & Araliaceae & Schefflera arboricola (Hayata) Merr. & cheflera \\
\hline 9 & Arecaceae & Phoenix canariensis hort. ex Chabaud & palmera canaria \\
\hline 10 & Arecaceae & Adonidia merrillii Becc. & palma de manila, palma de navidad \\
\hline 11 & Arecaceae & $\begin{array}{l}\text { Washingtonia filifera (Linden ex André) H.Wendl. } \\
\text { ex de Bary }\end{array}$ & palmera de california \\
\hline 12 & Asparagaceae & Agave americana $\mathrm{L}$. & agave, maguey, pita \\
\hline 13 & Asparagaceae & Chlorophytum comosum (Thunb.) Jacques & mala madre, lazo de amor \\
\hline 14 & Asparagaceae & Sansevieria trifasciata Prain & lengua de tigre, lengua de suegra \\
\hline 15 & Asteraceae & Senecio leucostachys Baker & viravira, huirahuira \\
\hline 16 & Asteraceae & Sonchus oleraceus L. & diente de león, amargón \\
\hline 17 & Bignoniaceae & Spathodea campanulata P. Beauv. & tulipán africano \\
\hline 18 & Bignoniaceae & Tecoma stans (L.) Juss. ex Kunth & huaranguay, tecoma \\
\hline 19 & Boraginaceae & Heliotropium angiospermum Murray & cola de alacrán, rabo de alacrán \\
\hline 20 & Cactaceae & Opuntia ficus-indica (L.) Mill. & tuna \\
\hline 21 & Caricaceae & Carica papaya $\mathrm{L}$. & papayo, papaya, papayero. \\
\hline 22 & Commelinaceae & Tradescantia pallida (Rose) D.R.Hunt & amor de hombre, purpurina \\
\hline 23 & Convolvulaceae & Ipomoea purpurea (L.) Roth & campanilla, auroras \\
\hline 24 & Crassulaceae & Kalanchoe delagoensis Eckl. \& Zeyh. & madre de miles \\
\hline 25 & Crassulaceae & Crassula argentea Thunb. & árbol de Jade \\
\hline 26 & Cupressaceae & Thuja occidentalis L. & árbol de la vida, tuya occidental \\
\hline 27 & Didiereaceae & Portulacaria afra Jacq. & $\begin{array}{l}\text { portulacaria, arbusto elefante, arbusto } \\
\text { japonés }\end{array}$ \\
\hline 28 & Euphorbiaceae & Acalypha wilkesiana Müll.Arg. & acalifa \\
\hline 29 & Euphorbiaceae & Codiaeum variegatum (L.) Rumph. ex A. Juss. & crotón \\
\hline 30 & Euphorbiaceae & Euphorbia candelabrum Tremaut ex Kotschy & candelabro, euforbia cactus \\
\hline 31 & Euphorbiaceae & Euphorbia lactea Haw. & planta candelabro \\
\hline 32 & Euphorbiaceae & Euphorbia pulcherrima Willd. ex Klotzsch & flor de pascua, pastora, buenas noches. \\
\hline 33 & Fabaceae & Acacia macracantha Humb. \& Bonpl. ex Willd. & huarango, faique, espino \\
\hline 34 & Fabaceae & Caesalpinia spinosa (Molina) Kuntze & tara, espino \\
\hline 35 & Fabaceae & Delonix regia (Bojer ex Hook.) Raf. & ponciana \\
\hline 36 & Fabaceae & Erythrina speciosa Andrews & árbol coral \\
\hline 37 & Fabaceae & Inga feuilleei DC. & pacae, pacay, huaba \\
\hline 38 & Fabaceae & Leucaena leucocephala (Lam.) de Wit & liliaque, yaravisco, chapra \\
\hline 39 & Fabaceae & Parkinsonia aculeata L. & palo verde, azote de cristo. \\
\hline 40 & Fabaceae & Acacia saligna (Labill.) Wendl. & mimosa, acacia azulada \\
\hline 41 & Geraniaceae & Pelargonium peltatum (L.) L'Hér. & geranio de hiedra, geranio \\
\hline 42 & Geraniaceae & Pelargonium $\times$ hortorum L.H. Bailey & geranio \\
\hline 43 & Lamiaceae & Salvia leucantha Cav. & salvia morada \\
\hline 44 & Lauraceae & Persea americana Mill. & palto, palta, aguacate \\
\hline 45 & Lythraceae & Punica granatum L. & granada \\
\hline
\end{tabular}


Continuación de Tabla 1

\begin{tabular}{|c|c|c|c|}
\hline$\overline{\mathbf{N}^{\circ}}$ & Familia & Nombre científico & Nombre común \\
\hline$\overline{46}$ & Malvaceae & Hibiscus rosa-sinensis L. & cucarda, rosa china \\
\hline 47 & Malvaceae & Malvaviscus arboreus Cav. & farolito \\
\hline 48 & Moraceae & Ficus carica L. & higuera, higo \\
\hline 49 & Moraceae & Ficus nitida Thunb. & ficus \\
\hline 50 & Moraceae & Morus nigra L. & mora, morera \\
\hline 51 & Musaceae & Musa x paradisiaca $\mathrm{L}$. & plátano, banano, cambur \\
\hline 52 & Myrtaceae & Callistemon lanceolatus (Sm.) Sweet & calistemo, escobillón, árbol del cepillo \\
\hline 53 & Myrtaceae & Eucalyptus globulus Labill. & eucalipto \\
\hline 54 & Nyctaginaceae & Bougainvillea spectabilis Willd. & buganbilia, buganvilla, papelillo \\
\hline 55 & Oleaceae & Jasminum officinale L. & jazmín, jazmín blanco. \\
\hline 56 & Pinaceae & Pinus pinea $\mathrm{L}$. & pino piñonero \\
\hline 57 & Plantaginaceae & Antirrhinum majus L. & doguito, perrito \\
\hline 58 & Proteaceae & Grevillea robusta A. Cunn. ex R.Br. & $\begin{array}{l}\text { grevillea, roble australiano, árbol de fuego o pino } \\
\text { de oro }\end{array}$ \\
\hline 59 & Rosaceae & Cotoneaster pannosus Franch. & cotoneaster plata \\
\hline 60 & Rosaceae & Prunus persica (L.) Batsch & duraznero, melocotonero \\
\hline 61 & Rosaceae & Rosa sp. & rosa \\
\hline 62 & Rutaceae & Citrus reticulata Blanco & mandarina \\
\hline 63 & Salicaceae & Populus deltoides Marshall & $\begin{array}{l}\text { chopo americano, álamo carolino, chopo de } \\
\text { virginia }\end{array}$ \\
\hline 64 & Sapotaceae & Pouteria lucuma (Ruiz \&Pav.) Kuntze & lúcuma, lucma \\
\hline 65 & Scrophulariaceae & Myoporum laetum $\mathrm{G}$. Forst. & mioporo, árbol ngaio \\
\hline 66 & Solanaceae & Brugmansia arborea (L.) Steud. & floripondio, campachu \\
\hline 67 & Solanaceae & Nicotiana glauca Graham & tabaco, tabaco silvestre \\
\hline 68 & Solanaceae & Physalis peruviana $\mathrm{L}$. & aguaymanto, capulí \\
\hline 69 & Solanaceae & Solanum peruvianum L. & tomate silvestre \\
\hline 70 & Xanthorrhoeaceae & Aloe vera (L.) Burm.f. & sábila, sávila, aloe de barbados o aloe de curazao \\
\hline
\end{tabular}

\subsection{Cuadros de especies}

Se realizaron fichas botánicas (Figura 2) para 65 especies, de las cuales 11 son especies endémicas del Perú (Tabla 2). Las especies Acacia macracantha (huarango), Parkinsonia aculeata (palo verde), Caesalpinia spinosa (tara), Nicotiana glauca (tabaco silvestre), Solanum pe- ruvianum (tomate silvestre), Sonchus oleraceus (diente de león) y Heliotropium angiospermum (cola de alacrán) están registradas como endémicas para las lomas costeras del Perú. Así mismo, estas especies han sido cultivadas en el área de estudio con fines ornamentales.

Tabla2. Lista de especies endémicas del Perú registradas en las áreas verdes del Centro Nuclear Oscar Miró Quesada de la Guerra (RACSO), Carabayllo. Lima.

\begin{tabular}{|c|c|c|c|}
\hline $\mathbf{N}^{\circ}$ & Familia & Nombre científico & Nombre común \\
\hline 1 & Anacardiaceae & Schinus molle L. & $\begin{array}{l}\text { molle serrano, árbol de la pimienta, árbol de la } \\
\text { vida. }\end{array}$ \\
\hline 2 & Annonaceae & Annona cherimola Mill. & chirimoya \\
\hline 3 & Bignoniaceae & Tecoma stans (L.) Juss. ex Kunth & huaranguay, tecoma. \\
\hline 4 & Fabaceae & Acacia macracantha Humb. \&Bonpl. ex Willd. & huarango, faique, espino. \\
\hline 5 & Fabaceae & Caesalpinia spinosa (Molina) Kuntze & tara, espino. \\
\hline 6 & Fabaceae & Inga feuilleei DC. & pacae, pacay, huaba. \\
\hline 7 & Sapotaceae & Pouteria lucuma (Ruiz \&Pav.) Kuntze & lúcuma, lucma. \\
\hline 8 & Solanaceae & Brugmansia arborea (L.) Steud. & floripondio, campachu. \\
\hline 9 & Solanaceae & Nicotiana glauca Graham & tabaco, tabacosilvestre. \\
\hline 10 & Solanaceae & Physalis peruviana $\mathrm{L}$. & aguaymanto, capulí. \\
\hline 11 & Solanaceae & Solanum peruvianum L. & tomate silvestre \\
\hline
\end{tabular}




\section{Discusión}

Como se esperaba, las especies encontradas en las áreas verdes del Centro Nuclear "Oscar Miró Quesada de la Guerra (RACSO)", pertenecen a familias y especies presentes en las áreas verdes de la ciudad de Lima así como en el Jardín Botánico Octavio Velarde Núñez de la UNALM [7, 2]. Esto facilitó su identificación sin lugar a dudas.

Las especies Acacia macracantha (huarango), Parkinsonia aculeata (palo verde), Caesalpinia spinosa (tara), Nicotiana glauca (tabaco silvestre), Solanum peruvianum (tomate silvestre), Sonchus oleraceus (diente de león) y Heliotropium angiospermum (cola de alacrán) han sido reportadas para las lomas de Villa María, lomas Lúcumo y lomas de Lachay $[11,12,13,14]$ y su presencia en el área estudiada indicaría que son especies ya adaptadas e introducidas en la jardinería de las áreas verdes de nuestra capital con cierta abundancia.

En un tiempo en que la ciudad de Lima Metropolitana lucha denodadamente por tener más metros cuadrados de áreas verdes por habitante frente a la creciente urbanización de los terrenos de la ciudad, el Centro Nuclear del IPEN se ha convertido en un lugar donde hay veces por encima del valor sugerido por la organización Mundial de la Salud que es de $9 \mathrm{~m}^{2}$ de áreas verdes por persona [6]. Esta situación hace del Centro Nuclear un lugar saludable para la vida humana.

Está ampliamente aceptado en el mundo entero que las áreas verdes dentro de las ciudades tienen una serie de efectos favorables sobre la vida de las personas; contribuyen a la conservación de la biodiversidad urbana (flora, aves, insectos, reptiles, mamíferos), regulan la temperatura ambiental y reducen los efectos de la luz solar mediante la formación de sombras (sombreamiento) y evapotranspiración. Asimismo, cohesionan el suelo, retienen la humedad y permiten la infiltración de aguas de lluvias, alimentando las napas subterráneas, entre otros beneficios [1].

El presente trabajo es un paso inicial en el esfuerzo que debe emprender nuestra institución para poner en valor el hermoso ecosistema dentro del cual estamos ubicados, convirtiéndolo en un lugar interesante, limpio, ordenado y apto para ser visitado y recorrido no solo por eventuales visitantes del Centro Nuclear, sino por los mismos trabajadores que podrían encontrar en las áreas verdes un lugar para conectarnos con la naturaleza, conocerla, cuidarla y disfrutarla en favor de nuestra propia salud emocional. El Centro Nuclear está ubicado en una privilegiada zona rural cerca de la gran Lima, donde sobra el aire puro y un terreno (mayormente eriazo) de 125 hectáreas que sobradamente podría albergar un jardín botánico y un campo agrícola experimental para el desarrollo de proyectos de investigación agropecuaria con universidades e instituciones nacionales y extranjeras.

\section{Conclusiones}

Se identificaron un total de 70 especies de plantas ubicadas dentro de las 16 hectáreas del Centro Nuclear "Oscar Miró Quesada de la Guerra (RACSO)" pero quedan aún muchas más por ser identificadas taxonómicamente tanto dentro de las 16 hectáreas como en las zonas aledañas, los cerros y el monte ribereño influenciado por el río Chillón.

Las fichas de información botánica colocadas en los carteles informativos en los jardines, pretenden despertar el interés de los trabajadores del IPEN en las plantas que están en contacto con ellos permanentemente pero que muchos no aprecian ni valoran. La confección de estas fichas busca convertir las áreas verdes del Centro Nuclear en una fuente permanente de información y acercamiento de los trabajadores a la naturaleza circundante.

El presente trabajo se circunscribe al inventario que se realizó dentro de las 16 hectáreas del Centro Nuclear siendo necesario realizar un inventario de la flora de los alrededores así como las zonas colindantes más allá de las 16 hectáreas.

\section{Agradecimientos}

A la Mg. Mercedes Flores Pimentel, jefa del Herbario MOL-Weberbauer de la Universidad Nacional Agraria La Molina, por su colaboración en la identificación de algunas especies. También expresamos nuestra gratitud al $\mathrm{Sr}$. Marino Rosales del IPEN por su apoyo en la ubicación e identificación de los nombres comunes de las plantas.

\section{Bibliografía}

[1] Reyes, S. 2012. Áreas verdes urbanas. Instituto de Estudios Urbanos y Territoriales Pontificia Universidad Católica de Chile. Disponible en http://www.ungs. edu.ar/ms ico/wp- $\quad$ content/uploads/2012/07/5.2Biodiversidad- urbana Sonia-Reyes-Paecke.pdf

[2] Enverdece Lima. Fondo Nacional del Ambiente Perú - FONAM. Disponible en http://www.fonamperu. org/general/document enverdece\%20Lima.p $\mathrm{df}$

[3] Guía de flora de las lomas costeras de lima. 2013. Ministerio de Agricultura (MINAG).

[4] Evaluación de la zona de emplazamiento y del área de influencia del centro nuclear de investigaciones del Perú. C.N.E.A. Comisión Nacional de Energía Atómica. República de Argentina. I.P.E.N. Instituto Peruano de Energía Nuclear. República Peruana. 1981.

[5] Savile, D. B. O. 1962. Collection and Care of Botanical Specimens. March 1962. Reprinted with Addenda 1973.Publication 1113. Research Branch, Canada Department of Agriculture.

[6] Guía virtual - Arboles en Lima. 2013. Disponible en http://es.scribd.com/doc/218379489/Guia- virtualArboles-en-Lima 
[7] García. C. y Gastañeta, M. 2008. Árboles de Lima. $1^{\circ}$ Edición. Lima-Perú.

[8] Brako, L. y Zarucchi, J. 1993. Catálogo de las Angiospermas y Gimnospermas del Perú. Missouri Botanical Garden.

[9] Ceroni, A. (-------). Diversidad de Angiospermas. Universidad Nacional Agraria La Molina. Facultad de Ciencias- Departamento de Biología.

[10] Flores, M. y Vilcapoma, G. 2006. Diversidad de Angiospermas. Guía de prácticas. Universidad Nacional Agraria La Molina. Facultad de Ciencias-Departamento de Biología.

[11] Trinidad, H. 2012. Flora vascular de las lomas de Villa María y Amancaes, Lima, Perú.

[12] Cano, A., Roque, J., Arakaki, M. et al. 1999. Diversidad florística de las lomas de Lachay (Lima) Durante el evento "El Niño 1997-98". Rev. Per.Biol. Vol. extraordinario: 125- 132.

[13] Strengthening Parks to Safeguard Biodiversity. ParksWatch. 2003. Perfil de Área Protegida - Perú. Reserva Nacional de Lachay. Disponible en www. parkswatch.org

[14] Cossíos, D., Valle, D., Castillo A. et al. 2002. Plan maestro de la Reserva Nacional de Lachay. 2003-2007. Instituto Nacional de Recursos Naturales (INRENA). 